\title{
Arbeit außerhalb der Arbeitswelt - neue Organisationen im Rahmen sozialer Bewegungen am Beispiel Spaniens
}

\author{
Ruth Simsa ${ }^{1}$
}

Online publiziert: 18. April 2016

(C) The Author(s) 2016. This article is available at SpringerLink with Open Access

Zusammenfassung Auf Basis von empirischer Feldforschung in Spanien diskutiert dieser Artikel organisationale Charakteristika von sozialen Bewegungsorganisationen. Soziale Bewegungsorganisationen gewinnen in quantitativer und qualitativer Hinsicht an Bedeutung. Sie haben häufig präfigurativen Charakter, d. h. sie versuchen, organisationalen Strukturen und Prozesse den angestrebten Zielen entsprechend zu gestalten. Der Artikel schließt mit Überlegungen, was aus den Experimenten und der Erfahrung dieser untypischen, sehr partizipativen und wenig hierarchischen Organisationen für die Führung und das Management auch von konventionellen Organisationen gelernt werden kann.

Schlüsselwörter Soziale Bewegungen · Präfigurative Organisationen · Bewegungsorganisation · organisatinales Lernen

The new world of work in Social Movements. The Spanish Case

Abstract Based on empirical field work in Spain, the paper discusses organizational features of social movement organizations and discusses, what conventional organizations and their leaders could be learn from them. It is argued that social movement organizations are gaining importance

a.o.Univ.Prof. R. Simsa

ruth.simsa@wu.ac.at

1 Institut für Soziologie und empirische Sozialforschung, Kompetenzzentrum für Nonprofit Organisationen und Social Entrepreneurship, Wirtschaftsuniversität Wien, Vienna University of Economics and Business, Welthandelsplatz 1, 1020 Wien, Österreich in both quantitative and qualitative terms. Given their prefigurative approach, a high level of experimentation with organizational forms is to be seen, resulting in decentralized, segmentary and group-based structures, which attempt at both a high level of inclusion and open membership and egalitarian, nonhierarchical processes. It is argued, that these structures, which resemble anarchist organizational theory and aim at a high degree of freedom and selfregulation require a high level of attention for communication structures, the clear focus on organizational values and ambiguity tolerance.

Keywords social movements - movement organizations · prefigurative organizations · organizational learning

\section{Bewegungsorganisationen - eine Neue Arbeitswelt mit Lernchancen}

Für viele Menschen spielt sich eine Neue Arbeitswelt außerhalb der Sphäre der Erwerbsarbeit $\mathrm{ab}$, nämlich in Organisationen sozialer Bewegungen $\left(\mathrm{SMO}^{1}\right)$, die sich zumeist als präfigurativ verstehen, also den Anspruch haben, durch neue Organisationsformen gesellschaftspolitische Ziele im Hier und Jetzt zu leben. Angesichts wachsender Arbeitslosigkeit und Exklusion sowie der Zunahme zivilgesellschaftlichen Engagements werden diese an Bedeutung zunehmen.

Die meisten SMO lehnen Hierarchie und herkömmliche Führung ab, sie können nicht auf finanzielle Incentives und übliche Motivationsstrategien zurückgreifen, weder gibt es ausgefeilte Businesspläne oder sonstige Managementtools, noch differenzierte formale Strukturen. Dennoch funktio-

${ }^{1}$ Die englische Abkürzung für social movement organizations hat sich durchgesetzt und wird daher auch hier verwendet. 
nieren viele der untersuchten Organisationen - entgegen allen Prämissen der Organisations- und Managementlehre erstaunlich gut. Angesichts der bestehenden Hierarchiekrise formaler Organisationen (Heintel und Krainz 2011) liegt die Frage nahe, was aus den Experimenten im Rahmen sozialer Bewegungen auch für herkömmliche Organisationen gelernt werden könnte. Ziel dieses Artikels ist somit neben der Darstellung der organisationalen Gemeinsamkeiten von SMO der spanischen Protestbewegung die Analyse möglicher Implikationen für Management, Führung und Beratung herkömmlicher Organisationen.

In Abschn. Hintergründe der wachsenden Bedeutung sozialer Bewegungsorganisationen werden zunächst die Hintergründe der Bedeutung von SMO kurz dargestellt: zunehmende Exklusion, die gegenwärtig wieder wachsende Bedeutung sozialer Bewegungen sowie die Krise herkömmlicher Organisationen. In Abschn. Die Organisation(en) des Protests in Spanien werden organisationale Strukturen und Prozesse im Rahmen der spanischen Protestbewegung beschrieben. Abschließend wird in Abschn. Lernchancen für konventionelle Organisationen? diskutiert, welche Erkenntnisse sich daraus für Wirtschaftsunternehmen ergeben könnten.

Empirisch gründet der Artikel auf Feldforschung zwischen Herbst 2014 und Sommer 2015. Neben teilnehmenden Beobachtungen bei Versammlungen, Essensausgaben oder Protestaktivitäten wurden 70 Interviews mit AktivistInnen und ExpertInnen in Madrid, Sevilla und Valencia durchgeführt. Die Zusammensetzung des Samples folgte Prinzipien der grounded theory, Datengewinnung und Analyse bzw. Kategorienbildung erfolgten also simultan, wobei ein möglichst heterogenes sample angestrebt wurde. Die Befragten waren zwischen 21 und 75 Jahre alt, hatten sehr unterschiedliche Bildungs- und Berufssituationen (Akademiker wie z. B. Universitätsprofessoren oder Anwälte, Arbeitslose, Studierende, Personen ohne formale Ausbildung etc.), Frauen waren leicht unterrepräsentiert (27). Es waren mehrheitlich AktivistInnen von Städten vertreten, allerdings auch einige aus ländlichen Gebieten. Die Befragten vertraten sehr unterschiedliche Organisationen, etwa Jugendinitiativen, Protestgroßeltern, Fraueninitiativen, die Plattform für Hypothekenbetroffene, Rechtsberatung, Stadtteilgruppen etc. Die verwendete Methode war das problemzentrierte Interview (Witzel 2000). 54 Interviews wurden aufgenommen, transkribiert und kodiert.

\section{Hintergründe der wachsenden Bedeutung sozialer Bewegungsorganisationen}

\subsection{Arbeit außerhalb der Arbeitswelt wird zunehmend zur Lebensrealität}

Bei Arbeitslosenzahlen von 2014 durchschnittlich um die $10 \%$ in Europa und einer Jugendarbeitslosigkeit von insgesamt über $21 \%$, mit Spitzenwerten in Griechenland $(50,7 \%)$ und Spanien $(53,7 \%)$ (European Commission, 2014b) bleiben die Arbeitswelt und ihre Organisationen vielen Menschen verschlossen. Damit ist das herkömmliche Bild von Organisationen schon jetzt für viele - v. a. junge - Menschen nicht mehr die Norm und es wird auch gesellschaftlich an Bedeutung verlieren. Gleichzeitig entstehen u. a. im Rahmen sozialer Bewegungen neue, unkonventionelle Organisationen. Im Sinne einer bewusstenschöpferischen Auseinandersetzung des Menschenmit der Naturund der Gesellschaftwird dort gearbeitet, oft in einem Stundenausmaß, das vielen Erwerbsarbeitsverhältnissen um nichts nachsteht.

Junge Menschen wenden sich also - freiwillig oder unfreiwillig - zunehmend von formalen Organisationen und auch traditionellen Formen von NPOs ab. Sie stehen vor der Herausforderung, kohärente Lebenswege in zunehmend flexiblen und unsicheren Kontexten zu entwickeln (Higgins und Porcaro 2013; Harris et al. 2010; Davis und McAdam 2000). Neue Formen von Organisation können somit im Sinn von Etzioni (1968, p. 121 und 154) als „creative responsiveness" gesehen werden.

\subsection{Die zunehmende Bedeutung sozialer Bewegungen und ihrer Organisationen}

Soziale Bewegungen haben immer schon organisationale Formen und Praktiken genutzt, um ihre Ziele zu erreichen. Gegenwärtig gewinnt dieses Phänomen wieder an Bedeutung. Es gibt eine massive Zunahme von zivilgesellschaftlichem Protest, von Aktivismus und freiwilliger Selbstorganisation. In den Krisenländern der EU sind regelmäßig Millionen Menschen auf den Straßen. Occupy und der Arabische Frühling wie auch Proteste in der Türkei hatten hohe Mobilisierungseffekte und brachten spezifische Organisationen hervor. 2015 hat die Zivilgesellschaft in Deutschland und Österreich wesentliche Beiträge zur Bewältigung der Flüchtlingskrise durch flexible und effektive, in unterschiedlichem Grad formalisierte Selbstorganisationen geleistet.

Das letzte Jahrzehnt wird insgesamt als Phase weltweiter und umfassender Mobilisierung gekennzeichnet (Kaldor und Selchow 2013; Anheier 2013; Benski et al. 2013; Graeber 2011). Es wird argumentiert, dass gegenwärtige $\mathrm{Ge}-$ sellschaften als ,social movement societies“ gesehen wer- 
den können, charakterisiert durch die wachsende quantitative und qualitative Bedeutung von Protest (Quaranta 2014; Meyer und Tarrow 1998) SMO sind somit erstens ein quantitatives Phänomen. Sie sind zweitens eine wichtige Voraussetzung der Effektivität und Nachhaltigkeit sozialer Bewegungen (Keck und Sikkink 1998; Simsa 2013; Tarrow 2001). Sie sind drittens auch qualitativ insofern bedeutsam, als sich viele Akteure sozialer Bewegungen in ihrer Identität und ihren Zielen explizit auf neue Formen von Organisation beziehen. Hier manifestiert sich vielfältiger Widerstand gegen managerialistische Diskurse (Spicer und Böhm 2007, 1691), etablierte Institutionen werden häufig ebenso abgelehnt wie starke Führung und formale Strukturen (Castells 2012; Hardt und Negri 2011; Della Porta und Diani 2006). Die Bewegungsforschung konstatiert folglich einen Fokus auf Experimente und die Entwicklung neuer Aktionsformen (Roth 2012). Nicht nur, wie einst, das Private, auch das Organisationale wird demnach als politisch gesehen.

Soziale Bewegungen selbst sind seit Jahren ein wichtiges Forschungsfeld (für einen Überblick siehe Della Porta und Diani (2015) sowie Roth und Rucht (2008)). Spezifische Forschungsfragen bezogen sich auf Mobilisierungsstrategien (Klandermans und Oegema 1987; Klandermans et al. 2014), Framing (Benford und Snow 2000; Snow et al. 1986), ihre kollektive Identität (Polletta und Jasper 2001; Flesher Fominaya 2010), ihre Beziehung zum Staat und anderen Institutionen (Tilly 2005), ihre Effekte (Earl 2000), ihre Dynamiken (Graeber 2011), Prozesse der Konstruktion von Sinn (Kurzman 2008; Melucci 1996), ihre Netzwerke (Castells 2012) und ihren Umgang mit Transnationalität (della Porta und Mattoni 2014).

Weder in der Organisationstheorie noch in der Bewegungsforschung wurden allerdings SMO hinreichend behandelt (Maeckelbergh 2011). Dies kann mit dem Vorherrschen des managerialistischen Diskurses zusammen hängen, welcher die Beobachtung von Organisationen eng mit den Imperativen von Hierarchie, Kontrolle und ökonomischer Instrumentalität verknüpft (Reedy 2014). Organisationstheorie ist überwiegend an Wirtschaftsorganisationen orientiert. Alternative Formen werden folglich eher vernachlässigt. Das ist historisch und feldspezifisch erklärlich. Die Bewegungsforschung interessiert sich für nicht-routinierte, bewegliche, radikale Aktivitäten mit dem Fokus auf Veränderung (Zald 2008), im herkömmlichen Bild von Organisation stehen Stabilität, rationale Kontrolle und Planbarkeit im Vordergrund (de Bakker et al. 2013, 575 f). Erst in jüngster Zeit ist das theoretische Interesse an SMO gestiegen (de Bakker et al. 2013; Laamanen und den Hond 2015; Den Hond et al. 2015; Walker 2012)

Auch in Bezug auf Bestrebungen, gesellschaftliche Bezüge wieder mehr auch in die Organisationstheorie zu bringen (Friedland und Alford 1991; Mikl-Horke 2011) und damit der Interpenetration von Organisationen und Gesell- schaft mehr Aufmerksamkeit zu schenken, sind SMO ein spannendes Phänomen. Im Sinne von ,,prefigurative politics“ streben sie häufig eine experimentelle Aktualisierung politischer Ideen im „Hier und Jetzt“ an (van de Sande 2013, 230). In der organisationalen Form sollen sich also Ansprüche an die Gesellschaft niederschlagen.

\subsection{Die Hierarchie- und Legitimationskrise herkömmlicher Organisationen}

Viele Wirtschaftsorganisationen aber auch NPOs sind im Stress. Spätestens seit der Wirtschaftskrise leiden viele unter finanziellen Problemen. Aufgrund massiven Kostendrucks wird häufig seit Jahren kaum Personal nachbesetzt und die vorhandene - oft gemeinsam alternde - Belegschaft wird zunehmend gefordert. Bei aller Verschiedenheit von Branchen und Organisationen werden sie generell als immer stärker getrieben beschrieben, Komplexität und Veränderungstempo nehmen $\mathrm{zu}$, die Wertschätzung für und Handlungsspielräume von Menschen hingegen ab. Schlagworte wie globale Vernetzung, steigender Wettbewerb, Kurzfristigkeit aufgrund der Kapitalmarktorientierung, steigender Kostendruck aufgrund der Finanzkrise und der schwachen europäischen Konjunktur werden auf vielen Arbeitsplätzen als konkrete Realität spürbar.

In dieser Situation wird auch das System der Hierarchie als nicht immer adäquat beschrieben. Auch wenn klassisch hierarchische Organisationen der Gesellschaft ungeahnte Effizienz und enorm gesteigerte Vermögen in Bezug auf Ressourcen und Produktivität beschert haben, haben sie ihre Schattenseiten. Unter dem Schlagwort „Hierarchiekrise" (Heintel und Krainz 2011) lassen sich ihre inhärenten Nachteile fassen: Hierarchien sind behäbig, haben zu wenig Reaktionsfähigkeit auf komplexe Problemlagen, sie dulden keinen Widerspruch - selbst wenn er funktional wäre sie führen oft zu mangelnder Integration der Gesamtorganisation, und geringe Partizipation an Entscheidungen führt häufig zu Widerständen und Demotivation. Zudem kenn ein Verlust von Legitimation und Moral konstatiert werden. Ortmann bezeichnet Organisationen als Orte normaler moralischer Katastrophen, als Moralverdrängungsmaschinen und Legitimationsfabriken (Ortmann 2010).

\section{Die Organisation(en) des Protests in Spanien²}

\subsection{Die Bewegung der ,Empörten“6}

Spanien ist eines der Länder, die von der Wirtschaftskrise am meisten betroffen sind. Die Lebensbedingungen haben sich in Folge enorm verschlechtert (Santamaría 2011,

\footnotetext{
${ }^{2}$ Mit Dank an Marion Totter für anregende Diskussionen.
} 
21). So lag im Jahr 2014 die Arbeitslosigkeit bei 26,38\% ${ }^{3}$ und die Jugendarbeitslosigkeit war mit 53,5\% die höchste in Europa ${ }^{4}$, was viele von einer verlorenen Generation sprechen lässt. 2012 lebten 22,2 \% der Wohnbevölkerung unter der Armutsgrenze. Mehr als ein Drittel aller Kinder ist armutsgefährdet und von sozialer Exklusion bedroht (EU-SILC 2913; SavetheChildren 2014). Dies und die hohe Korruption führte zu einer massiven Vertrauenskrise in das politische System (Hughes 2011). Daran entzündete sich, beginnend mit Platzbesetzungen und Massenprotesten, im Jahr 2011 die Protestbewegung der „Indignados“, der Empörten (Simsa et al. 2015a). Die Bewegung war der Ausgangspunkt für weitere Politisierung und eine starke Zunahme zivilgesellschaftlicher Aktivitäten. Die Zahl der Protestaktivitäten stieg weiter an (García 2013, 303). In der Zivilgesellschaft „brodelt es“, es ist eine „explosive Situation“, ,,sehr energievoll und bewegt“. So beschreiben viele den dynamischen Zustand.

\subsection{Heterogene Organisationen mit ähnlichen Kernelementen}

Seit Mai 2011 gab es eine institutionelle Ausdifferenzierung von Engagement. Erstens gewannen „Mareas“ (zu dt.: Flut) an Bedeutung, die als eine Art themenorientierte „Bewegung in der Bewegung“ verstanden werden können, etwa die „Marea Azul“, die sich gegen die Privatisierung des Wassers ausspricht. Die Marea Granate verbindet die wegen der Krise ausgewanderten Jugendlichen. Zweitens gibt es eine Vielzahl an sehr unterschiedlichen Assoziationen, die im weitesten Sinn als normale zivilgesellschaftliche Organisation verstanden werden können, allerdings nur teilweise formalen Status haben. Besondere Legitimität genießen die landesweit vernetzten Organisationen gegen Zwangsräumungen, aber auch ,Jugend ohne Zukunft“, die „Protestgroßeltern“, Stadtteilversammlungen etc. Drittens sind sehr lose organisierte, kaum formal strukturierte aber dennoch langlebige Initiativen entstanden, wie z. B. „Zeitbanken“, bei denen Arbeitsleistungen getauscht werden, soziale Währungen oder private Essensausgaben. In einer dieser Initiativen in Madrid werden z. B. täglich bis zu 180 hungrige Personen von Freiwilligen mit Essen versorgt. Viertens ist die Bewegung im Jahr 2014 mit der Gründung der Partei „Podemos“, mit deren Erfolgen bei der Europawahl und der nachfolgenden Welle von Gründungen regionaler Parteien in eine neue Stufe der partiellen Institutionalisierung getreten (Feenstra und Keane 2014).

\footnotetext{
3 http://de.statista.com/statistik/daten/studie/17327/umfrage/ arbeitslosenquote-in-spanien/Zugriff 17.3.2015

${ }^{4}$ http://de.statista.com/statistik/daten/studie/74795/umfrage/ jugendarbeitslosigkeit-in-europa/Zugriff 17.3.2015
}

Bei aller organisationalen Heterogenität gibt es starke verbindende Elemente. Neben ähnlichen Zielen ist dies zunächst die operative Form der Organisation, die Asamblea. Der Begriff bedeutet „Versammlung“, meint im Rahmen der Bewegung allerdings Versammlungen, die dem Anspruch nach offen, egalitär, öffentlich, konsensbasiert und weitgehend unstrukturiert sind. Sie werden als das Instrument direkter Demokratie gesehen, durch Arbeitsgruppen vorbereitet und haben keine LeiterInnen, nur rotierende SprecherInnen (Madrilonia 2012). Auch wenn sie dem Anspruch nach völlig offen sind, werden sie meist durch einen Kern an aktiven Mitgliedern getragen.

Auch Orte haben hohe Bedeutung. Das Internet wird zwar stark genutzt, der zentrale identitätsstiftende Ort der Bewegung ist allerdings der öffentliche Raum, also die physische Anwesenheit auf Straßen und Plätzen. Die kollektive Aneignung des öffentlichen Raums hat in Spanien historische Tradition (Janoschka und Sequera 2011). Mit der Bewegung hat sich ihre Bedeutung wieder stark erhöht, in $\mathrm{Zu}$ sammenhang mit Demonstrationen und Camps, aber auch besetzten Häusern. Wie auch die Camps gelten diese als gelebte Praxis eines alternativen Gesellschaftsentwurfs, die dezentrale Organisation und Bedürfnisorientierung erprobt (Hughes 2011, 412).

Zentrale Grundsätze all dieser Organisationen entsprechen weitgehend jenen der anarchistischen Organisationstheorie (Graeber 2002; Reedy 2014), feministischen Prinzipien (Siltanen et al. 2014; Butler 1995) sowie auch den in vorangegangenen Bewegungen entwickelten, insbesondere im Antiglobalization movement (Flesher Fominaya 2014; Graeber 2011; Maeckelbergh 2012). Zusammengefasst sind das die Folgenden:

- Selbststeuerung und Egalität

Damit einher geht eine Ablehnung von Funktionsautorität, Hierarchie und jeglicher Form der Dominanz zugunsten von horizontalen Strukturen. In fast allen Interviews wird der Begriff der "Selbststeuerung“ als zentrales Moment der Identität genannt, oft noch vor den inhaltlichen Zielen. Egalität wird als Voraussetzung von Selbststeuerung bezeichnet - ohne diese wäre Selbststeuerung ein legitimatorischer, hohler Begriff. Selbststeuerung und egalitäre Strukturen werden als Ziel an sich gesehen, sowie als notwendige organisationale Voraussetzung für das Erreichen gesellschaftlicher Veränderung.

In Bezug auf den Anspruch der Vermeidung von Hierarchie gibt es viel an Diskussion sowie die Entwicklung von Praktiken und Prozessen zur Vermeidung informeller Hierarchien. Mit diesen soll Leadership auf unterschiedliche, wechselnde Personen verteilt und notwendige Führung gesichert werden: ,just because an organization is 
leaderless, it does not necessarily mean that it is also leadershipless" (Sutherland et al. 2013, 759).

- Offenheit und damit Partizipation möglichst vieler Personen

Die Mitgliedschaft ist oft lose und realisiert sich v. a. über gemeinsame Aktivitäten. Während klassische Organisationen über Mitgliedschaftsbedingungen definiert werden können, ist hier ein Kontinuum an Verbindlichkeit feststellbar. Mitgliedschaft und damit gewisse Entscheidungsbefugnisse gründen in den aus der Bewegung hervor gegangenen Parteien auf einer reinen Registrierung auf entsprechenden Internetplattformen, bei Mareas und auch manchen Asambleas sind sie durch bloße Anwesenheit gegeben und bei fokussierteren Initiativen gibt es klare, wenn auch sehr offen gehaltene Bedingungen. Mit weiterer Differenzierung im Lebenszyklus einzelner Organisationen wird die Frage der Offenheit voraussetzungsvoll und es stellt sich die Gefahr von Unterwanderung wie auch die Notwendigkeit der inhaltlichen wie auch technischen Weiterentwicklung von partizipativen Entscheidungsstrukturen. Dies hat v. a. die neuen Parteien befasst, deren Mitgliederzahl zu groß ist, um persönliche Beziehungen und Vertrauen als Basis von Strukturen gelten zu lassen.

- Dezentralisierung und segmentäre, netzwerkförmige Organisationen

Diese netzwerkförmigen Organisationen bestehen oft aus unterschiedlichen Gruppen, die polyzentrisch verbunden sind, also oft kein klares Steuerungszentrum haben. Sie entsprechen am ehesten dem System überlappender Gruppen (Likert 1967), welche über Doppelmitgliedschaften einzelner Personen verbunden sind. Zusammen gehalten werden sie zudem über Verfassungen, die zentrale Inhalte wie auch prozedurale Vorgangsweisen außer Streit stellen.

- Fluide und experimentelle Organisation

Die Organisation ist verhandelbar, kann jederzeit verändert werden, sie wird explizit nicht nur als Mittel zum Zweck gesehen, sondern als präfigurative Verkörperung der angestrebten Welt. Aus diesem Grund wird der hohe prozedurale Aufwand in der Regel akzeptiert. Es geht eben nicht nur um ein möglichst effizientes Erreichen eines außerhalb liegenden Ziels, sondern um dessen Realisierung - der organisationale „Weg“ selbst ist in den Augen der Akteure legitimes und sinnvolles Ziel.

- Geteilte Werte als zentrales Element der Identität Grundlage der vorangegangenen Aspekte sind Selbstbeschreibungen anhand geteilter Werte. Die Bewegung will sich zwar keiner politischen Richtung zuordnen, sie präsentiert sich als heterogen und inklusiv, vertritt aber Werte, die eher als „links“ gelten, wie Umverteilung, einen starken Sozialstaat oder Regulierungen des Finanzsystems. Die Wut auf eine korrupte politische und wirt- schaftliche Elite, die sogenannte „Kaste“, sowie fundamentale Kritik kapitalistischen Systems sind inhaltliche Basis der geteilten Werte. Der Slogan „Es ist keine Krise, es ist Kapitalismus“ bringt dies auf den Punkt und führt zur Entwicklung von inhaltlichen ,counter narratives" (Pianta 2013, 156).

Mindestens so wichtig, wie die konkreten Inhalte dieser Werte ist ihre hohe und von keinem Akteur in Frage gestellte Bedeutung dieser Werte als wesentliches Moment der Identität in Selbstbeschreibungen. Wertorientierung wird nicht als wünschenswerte Rahmenbedingung organisationaler Zielerreichung gesehen, sondern als fundamentaler Teil derselben. Zudem ist die Form der Organisation selbst inhaltlich wertorientierter Aspekt der Ideologie: ,Those new forms of organization are its ideology." (Graeber 2002, 70).

- Eine neue Balance des Widerspruchs von Person und Organisation

Den Werten entsprechend wird auch eine hohe Bedeutung der einzelnen Person, sozusagen des Menschen als Menschen statt als Funktionsträger, angestrebt. Einzelnen Personen und ihren Befindlichkeiten und Bedürfnissen wird ebenso Raum gegeben, wie auch der Austragung von persönlichen Konflikten.

In diesem Sinn ist die Bedeutung von Personen höher als in konventionellen Organisationen.

Sie ist gleichzeitig auch geringer, da sie nicht an Funktionsautorität, sondern unmittelbarer an die Passung der Fähigkeiten an die jewiligen Erfordernisse der Situation gebunden ist.

In dem Zusammenhang steht auch die hohe Bedeutung von Emotionen. Diese ist auch in konventionellen Organisationen gegeben, wird hier aber auf einer sehr expliziten Ebene ausgehandelt. Auf Versammlungen wird geweint, gestritten, umarmt und dies alles ohne großes Aufhebens. Dies ist nicht nur kulturspezifisch erklärlich, bei traditionellen NPOs ist dies deutlich weniger beobachtbar. Es ist vermutlich u. a. auf die zentrale Umwelt der Organisationen, die soziale Bewegung, zurückzuführen. Emotionen motivieren zur Mobilisierung und wurden von der Bewegung auch generiert (Benski 2011; Jasper 2011), zum einen durch Slogans (,wir haben keine Angst“; „Du brauchst Dich nicht zu schämen“ ...) zum anderen durch Praktiken der Vergemeinschaftung belastender Erfahrungen. Neben Wut und Enttäuschung wird oft auch der hohe Stellenwert von positiver Energie, Lust am Experimentieren, und Freude genannt („Wir haben die Lust am Organisieren entdeckt.“).

- Klare Regeln von Entscheidungsstrukturen und der Kommunikation:

In Selbstbeschreibungen heißt es gerne, die einzige Regel sei jene, dass es in diesen, sich oft als anarchistisch 
verstehenden Organisationen, keine Regel gäbe (Graeber 2011, 135). Sowohl theoretisch wie auch praktisch wird aber viel in die Entwicklung und Einhaltung von Entscheidungs- und Kommunikationsregeln investiert. Diese sind verhandelbar und bedürfen der Akzeptanz, werden aber zumeist strikt eingehalten. Beispiele sind etwa ein Reißverschlusssystem zwischen Männern und Frauen für Redebeiträge in Gruppen oder die Regel „,Kein Vorschlag ohne triftigen Grund“. Diese ist notwendig, da der Konsensprozess nur kombiniert mit dem Prinzip radikaler Dezentralisierung funktioniert: Entscheidungen sollten daher in möglichst kleinen Gruppen getroffen und nur in größere Gremien gebracht werden, wenn dies unabdingbar ist.

- Konfliktbereitschaft

Während im Gegensatz zu Bewegungskulturen der 60erJahre in den letzten Jahrzehnten oft auf Harmonie und Konfliktvermeidung gesetzt wurde, was nicht nur als dysfunktional sondern auch z. T. als repressiv empfunden wurde, wird nun die Sinnhaftigkeit von Konflikten betont und auch die Grenzen von Inklusion diskutiert.

\section{Lernchancen für konventionelle Organisationen?}

All dies ist voraussetzungsvoll und birgt Fallen, wie etwa den hohen Zeitaufwand, (siehe z. B. „Freedom is an Endless Meeting“, Polletta 2002). Dennoch scheint es erstaunlich gut zu funktionieren, gemessen an Selbstbeschreibungen, inhaltlichen Erfolgen ${ }^{5}$ und mittlerweile z. T. langjährigen Überleben der Organisationen. Was dies im Kern möglich macht, sind stark geteilte Werte, persönliche Beziehungen (Pickard, 2006, S. 320) zumindest der Kerngruppen und der starke Wunsch und die aktive Bereitschaft, zu experimentieren.

Kann daraus für das Management oder die Beratung herkömmlicher Unternehmen gelernt werden? Zum einen folgen diese natürlich völlig anderen Mustern und Zielen, zum anderen haben die Kulturmuster der SMO auch ihre Nachteile. Im Folgenden werden dennoch drei Anregungen genannt und mit jeweils einem für Wirtschaftskontexte entwickelten Modell ergänzt:

- Aufmerksamkeit und Kreativität in Bezug auf Kommunikationsstrukturen

Es wird in den SMO viel in die Entwicklung adäquater Kommunikationsstrukturen investiert und dies lohnt

\footnotetext{
${ }^{5}$ Der Plattform für Hypothekenbetroffene z. B. gelang es bis zum Jahr 2014, mehr als 1150 Zwangsräumungen zu verhindern, und für die Unterbringung von 1.180 Betroffenen zu sorgen (PAH http:// afectadosporlahipoteca.com/recursos-graficos/ 21.12.2014).
}

bekanntlich: „Ob die Intelligenz eines sozialen Systems größer oder kleiner ist als die seiner Mitglieder, hängt davon ab, wie Kommunikation organisiert ist." (Simon 2004, S. 12) Die enge Kopplung der Akteure mit relativer Offenheit von Aktionen wird ebenfalls oft als Erfolgsfaktor beschrieben (ebd., S. 111).

In der Wirtschaftswelt gibt es derzeit ebenfalls die Suche nach neuen Kommunikationsstrukturen, wie sie z. B. das Modell der Holacracy verspricht. Mit Fokus auf den Daseinszweck der Organisation regelt dieses die basalen $\mathrm{Re}$ geln des Zusammenspiels und der Entscheidungsfindung auf allen Ebenen der Organisation, basierend auf selbstorganisierten Kreisen mit Doppelmitgliedschaften, möglichst verteilter Autorität und einer Hierarchie von Wirkungsbereichen statt jener von persönlichen Funktionen (Simsa et al. 2015b). Das Modell weist viele Ähnlichkeiten zu Mustern der SMO auf. Generell stecken in der hohen Bedeutung von und dem Experimentieren mit Kommunikationsstrukturen in SMO hohe Lernchancen für BeraterInen und Führungskräfte.

- Zelte statt Paläste - Mut zu flexiblen Strukturen und einem gewissen Maß an Unklarheit

Angesichts unsicherer, komplexer und sich verändernder Umwelten braucht es u. U. mehr an struktureller Ambiguitätsoffenheit. In diese Richtung gehen Vorschläge, die Hedberg u. a. bereits 1976 auch für Wirtschaftsunternehmen gemacht haben (Hedberg et al. 1976). Statt „Palästen“, welche auf Spezialisierung, eindeutigen Zielen und klaren Autoritätsstrukturen, stabilen Verantwortlichkeiten, klaren Entscheidungskriterien und Routineprogrammen beruhen, welche v. a. in stabilen Umwelten erfolgreich sein können, sehen sie in eher veränderlichen Umwelten ,Zelte“ als geeignete Organisationsform. „Residents of changing environments need a tent" (ebd., S. 45). Diese betonen Flexibilität, Kreativität und Initiative mehr als Autorität und Klarheit. Sie verlangen weder Harmonie zwischen Aktivitäten einzelner Organisationsteile noch konsistentes Verhalten im Zeitablauf. Das Motto lautet: ,Why behave more consistently than one's world does?“" (Ebd., S. 45).

Die Autoren plädieren für ambigue Autoritätsstrukturen, unklare Ziele und Widersprüche und sogar Unentschiedenheit und verweisen auf empirische Befunde, dass diese die Resilienz in Krisen erhöhen. Entscheidend sind dabei intensive informelle Kommunikation, partizipative Strukturen und Entscheidungsmacht in dezentralen Einheiten. Statt auf festen Rollen und Funktionen liegt die Aufmerksamkeit auf Fähigkeiten und Beziehungen, welche zum Prozess der Generierung der ,organization to come“ beitragen. 
- Konsequente Orientierung an organisationalen Werten „Das Verhältnis von Organisation und Moral ist kein Liebesverhältnis.“ (Ortmann 2010, 72) Es kann aus strukturellen Gründen wohl auch keines sein. Werte und soziale Verantwortung spielen aber auch im Diskurs über die Wirtschaft eine zunehmende Rolle. Das ,historisch einzigartige Moralisierungsniveau“ (Weber 2011), wird von Unternehmen zunehmend mit Aktivitäten unter dem Label Corporate Social Responsibility (CSR) beantwortet. Wenn CSR-Aktivitäten zu Marketingzwecken eher den Charakter einer Inszenierung haben, kann dies zwar dennoch einen Wandel der gesellschaftlichen Rollendefinition von Unternehmen anregen (Curbach 2009), greift aber in Bezug auf Committment und Motivation von MitarbeiterInnen sicher zu kurz. Die hohe Bedeutung gelebter organisationaler Werte wird in den beschriebenen SMO deutlich. In Wirtschaftsunternehmen gibt es hier noch viel Luft nach oben.

Es mag sein, dass die „Zelte“ der spanischen und anderer sozialer Bewegungen gemessen an ihren wirtschaftlichen Beiträgen ein Randphänomen bleiben und sie haben auch ihre organisationalen Defizite. Einzelne Elemente dieser Organisationen sind aber vermutlich nachhaltiger als die Paläste der Industrie und Finanzwirtschaft, die an Sinnund Motivationsverlust leiden und deren Wachstumsmodell soziale und ökologische Probleme schafft. Wenn veränderliche Umwelten flexible Strukturen, gute soziale Netzwerke und Beziehungen, unerbittliche Orientierung am Sinn und am gesellschaftlichen Nutzen sowie effektive Kanalisierung von Fähigkeiten brauchen, dann findet man vieles davon in den SMO. Sie bieten damit Anregungen auch für konventionelle Organisationen, deren Führungskräfte und Berater. Möglicherweise kann damit der Slogan der Indignados, „Eine andere Welt ist möglich“ zumindest teilweise umgewandelt werden zu „Eine andere Organisation ist möglich“.

Open Access Dieser Artikel unterliegt den Bedingungen der Creative Commons Attribution License. Dadurch sind die Nutzung, Verteilung und Reproduktion erlaubt, sofern der/die Originalautor/en und die Quelle angegeben sind.

\section{Literatur}

Anheier, H. K. (2013). Entwicklungen der internationalen Zivilgesellschaft. In R. Simsa, M. Meyer, \& C. Badelt (Hrsg.), Handbuch der Nonprofit-Organisation. Strukturen und Management (S. 77-89). Stuttgart: Schäffer-Poeschel.

Benford, R.D., \& Snow, D. A. (2000). Framing processes and social movements: an overview and assessment. Annual Review of Sociology, 26, 611-639.

Benski, T. (2011). Emotion maps of participation in protest: The case of women in black against the occupation in Israel. Research in Social Movements, Conflicts and Change, 31, 3-34.
Benski, T., Langman, L., Perugorría, I., \& Tejerina, B. (2013). From the streets and squares to social movement studies: What have we learned? Current Sociology. La Sociologie Contemporaine, 61(4), $541-561$

Butler, J. (1995). Contingent foundations. In S. Benhabib, J. Butler, D. Cornell, \& N. Fraser (Hrsg.), Feminist contentions: a philosophical exchange (S. 35-58). New York: Routledge.

Castells, M. (2012). Networks of outrage and hope: Social movements in the internet age. Cambridge: Polity Press.

Curbach, J. (2009). Die Corporate-Social-Responsibility-Bewegung. Berlin: Springer.

Davis, G. F., \& McAdam, D. (2000). Corporations, Classes, and Social Movements After Managerialism. Research in Organizational Behavior, 22, 193-236.

de Bakker, F. G. A., den Hond, F., King, B., \& Weber, K. (2013). Social movements, civil society and corporations: taking stock and looking ahead. Organization Studies, 34(5-6), 573-593.

Della Porta, D., \& Diani, M. (2006). Social movements an introduction (2. Aufl.). Oxford: Blackwell.

Della Porta, D. (2015). Introduction. In D.Della Porta, \& M. Diani (Hrsg.), The oxford handbook of social movements (S. 1-31). Oxford: Oxford University Press.

Della Porta, D., \& Mattoni, A. (Hrsg.). (2014). Spreading protest. social movements in times of crisis. UK: ECPR Press.

Den Hond, F., De Bakker, F. G. A., \& Smith, N. (2015). Social movements and organizational analysis. In D.Della Porta, \& M. Diani (Hrsg.), The oxford handbook of social movements (S. 291-306). Oxford: Oxford University Press.

Earl, J. (2000). Methods, movements, and outcomes. Research in Social Movements, Conflicts and Change, 22, 3-25.

Etzioni, A. (1968). The Active Society. New York: Free Press.

Feenstra, R. A. (2014). Politics in spain: a case of monitory democracy. VOLUNTAS, 25(5), 1262-1280.

Flesher Fominaya, C. (2010). Collective identity in social movements: central concepts and debates. Sociology Compass, 4(6), 393-404.

Flesher Fominaya, C. (2014). Debunking spontaneity: spain's 15-M/ Indignados as autonomous movement. Social Movement Studies, 14(2), 142-163.

Friedland, R., \& Alford, R. (1991). Bringing society back. In W. Powell, \& P. Dimaggio (Hrsg.), Symbols, practices and institutional contradictions. New institutionalism in organizational analysis (S. 232-263). Chicago: University Of Chicago Press.. In The

García, O. J. M. (2013). Soft repression and the current wave of social Mobilisations in spain. Social Movement Studies, 13(2), 303-308.

Graeber, D. (2002). The new anarchists. New Left Review, 13, 61-73.

Graeber, D. (2011). Occupy Wall Street's anarchist roots. http://www. aljazeera.com/indepth/opinion/2011/11/2011112872835904508. html. Zugegriffen: 11.02. 2015

Hardt, M., \& Negri, A. (2011). The fight for ,real democracy ' at the heart of Occupy Wall Street. In Foreign Affairs. http://www. foreignaffairs.com/articles/136399/michael-hardt-and-antonionegri/the-fight-for-real-democracy-at-the-heart-of-occupy-wallstreet. Zugegriffen: 15.4.2015

Harris, A., Wyn, J., \& Younes, S. (2010). Beyond apathetic or activist youth. Young, 18(1), 9-32.

Hedberg, B., Bystrom, P.C. W.H., \& Starbuck, W.H. (1976). Camping on seesaws: prescriptions for a self-designing organization. Administrative Science Quarterly, 21(1), 41-65.

Heintel, P., \& Krainz, E.E. (2011). Projektmanagement Hierarchiekrise, Systemabwehr, Komplexitätsbewältigung (5. Aufl.). Wiesbaden: Gabler.

Higgins, J., \& Porcaro, G. (2013). From crisis to credibility: the need for quality jobs for young people. European View, 12(2), 189-198.

Hughes, N. (2011). Young people took to the streets and all of a sudden all of the political parties got old': the $15 \mathrm{M}$ movement in spain. Social Movement Studies, 10(4), 407-413. 
Janoschka, M., \& Sequera, J. (2011). Zur symbolischen Rückeroberung des öffentlichen Raums. Eine Analyse der Raumpolitiken des Movimiento 15-M. PROKLA, 166, 151-162.

Jasper, J. M. (2011). Emotions and social movements: twenty years of theory and research. Annual Review of Sociology, 37(1), 285-303.

Kaldor, M., \& Selchow, S. (2013). The ,bubbling up" of subterranean politics in europe. Journal of Civil Society, 9(1), 78-99.

Keck, M.E., \& Sikkink, K. (1998). Activists beyond bordersadvocacy networks in international politics. Ithaca, NY: Cornell Univ. Press.

Klandermans, B., \& Orgema, D. (1987). Potentials, networks, motivations, and barriers: steps towards participation in social movements. American Sociological Review, 52(4), 519-531.

Klandermans, B., van Stekelenburg, J., Damen, M.-L., van Troost, D., \& van Leeuwen, A. (2014). Mobilization without organization: the case of unaffiliated demonstrators. European Sociological Review, 30(6), 702-716.

Kurzman, C. (2008). Introduction: Meaning-making in social movements. Anthropological Quarterly, 81(1), 5-15.

Laamanen, M., \& den Hond, F. (2015). Prefigurative partial organization in local social movements: Examining decided and emergent order in a time bank. Athens: EGOS.

Likert, R. (1967). The human organization: its management and value. New York: McGraw-Hill.

Madrilonia, C. (2012). Cuando la gente reinventa la política. Lenguajes y actitudes del movimento 15 M. In J.C. Fernandez (Hrsg.), Ocupemos el mundo (S. 53-67). Sevilla.

Maeckelbergh, M. (2011). Doing is believing: Prefiguration as strategic practice in the Alterglobalization movement. Social Movement Studies, 10(1), 1-20.

Maeckelbergh, M. (2012). Horizontal democracy now: from Alterglobalization to occupation. Interface, 4(1), 207-234.

Melucci, A. (1996). Challenging codes: Collective action in the information age. Cambridge: Cambridge University Press.

Meyer, D. S., \& Tarrow, S. (1998). The social movement society: contentious politics for a new century. Washington: Rowman \& Littlefield.

Mikl-Horke, G. (2011). Sozioökonomie. Die Rückkehr der Wirtschaft in die Gesellschaft. Marburg: Metropolis.

Ortmann, G. (2010). Organisation und Moral. Die dunkle Seite. Marburg: Metropolis.

Pianta, M. (2013). Democracy Lost: The Financial Crisis in Europe and the Role of Civil Society. Journal of Civil Society, 9(2), 148-161.

Pickard, V. (2006). United Yet Autonomous: Indymedia and the Struggle to Sustain a Radical Democratic Network. Media, Culture and Society 28:315-336

Polletta, F. (2002). Freedom is an endless meeting: democracy in american social movements. Chicago: University of Chicago Press.

Polletta, F., \& Jasper, J. M. (2001). Collective identity and social movements. Annual Review of Sociology, 27, 283-305.

Quaranta, M. (2014). Collective and private resources and the inequalities of non-violent political protest in european countries. Journal of Civil Society, 10(3), 294-316.

Reedy, P. (2014). Impossible organisations: Anarchism and organisational praxis. Ephemera, 14(4), 639-658.

Roth, R. (2012). Vom Gelingen und Scheitern sozialer Bewegungen. Forschungsjournal Soziale Bewegungen, 1, 21-31.

Roth, R., \& Rucht, D. (Hrsg.). (2008). Die Sozialen Bewegungen in Deutschland seit 1945. Ein Handbuch. Frankfurt: Campus.

Santamaría, A. (2011). La rebelión de los Indignados. Reflexiones a pie de acampada. El Viejo Topo, 282/283, 20-25.

SavetheChildren (2014). Pobreza infantil y exclusión social en Europa. Una cuestión de derechos. Bd. 42. Brüssel: Save the Children.

Siltanen, J., Klodawsky, F., \& Andrew, C. (2014). „This is how I want to live my life": An Experiment in Prefigurative Feminist Organizing for a More Equitable and Inclusive City. Antipode, 47(1), 260-279.

Simon, F.B. (2004). Gemeinsam sind wir blöd!? Die Intelligenz von Unternehmen, Managern und Märkten. Carl-Auer-Systeme
Simsa, R. (2013). Protest ohne Organisation? Neue Soziale Bewegungen, 4, 24-38.

Simsa, R., Heinrich, M., \& Totter, M. (2015a). Von der Puerta del Sol ins Europaparlament. Organisationale Ausdifferenzierungen der spanischen Protestbewegung. Neue Soziale Bewegungen, 3, 8-17.

Simsa, R., Mitterer, G., Taudes, A., Leo, H., \& Seuhs-Schoeller, C. (2015b). Soziale Entscheidungsverfahren. In R. Eschenbach, C. Horak, M. Meyer, \& C. Schober (Hrsg.), Management der Nonprofit-Organisation. Stuttgart: Schaeffer Poeschel.

Snow, D. A., Rochford, E. B., Worden, S. K., \& Benford, R. D. (1986). Frame alignment processes, Micromobilization, and movement participation. American Sociological Review, 51(4), 464-481.

Spicer, A., \& Böhm, S. (2007). Moving management: theorizing struggles against the hegemony of management. Organization Studies, 28(11), 1667-1698.

Sutherland, N., Land, C.S., \& Böhm, S. (2013). Anti-leaders(hip) in Social Movement Organizations: The case of autonomous grassroots groups. Organization, 21:759-781.

Tarrow, S. (2001). Transnational politics: Contention and institutions in international politics. Annual Review of Political Science, 4, 1-20.

Tilly, C. (2005). Social Movements, 1768-2004. Boulder: Paradigm.

van de Sande, M. (2013). The Prefigurative politics of Tahrir square: an alternative perspective on the 2011 revolutions. Res Publica, 19(3), 223-239.

Walker, E.T. (2012). Social Movements, Organizations, and Fields: a Decade of Theoretical Integration. Contemporary Sociology, 41(5), 576-587.

Weber, A. T. F. (2011). Die Moralisierung der Wirtschaft. CSR als strategisches Element neoliberaler Gesellschaftspolitik? In G. MiklHorke (Hrsg.), Sozioökonomie. Die Rückkehr der Wirtschaft in die Gesellschaft (S. 367-400). Marburg: Metropolis.

Witzel, A. (2000). The problem-centered interview. Forum Qualitative Sozialforschung / Forum: Qualitative Social Research, 1(1).

Zald, M. N. (2008). Epilogue: Social Movements and Political Sociology in the Analysis of Organizations and Markets. Administrative Science Quarterly, 53(3), 568-574.

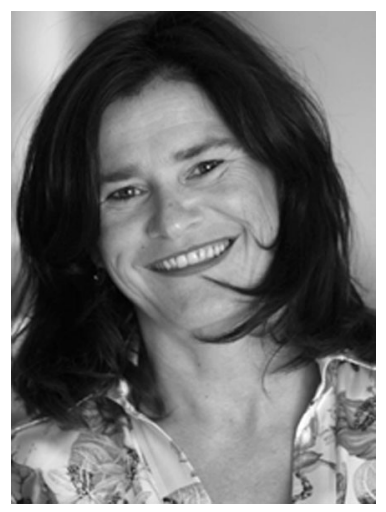

a.o.Univ.Prof. Ruth Simsa ist Professorin am Institut für Soziologie und empirische Sozialforschung sowie in der Leitung des Kompetenzzentrums für Nonprofit Organisationen und Social Entrepreneurship an der Wirtschaftsuniversität Wien. Sie hat langjährige Erfahrung als Organisationsberaterin und Führungskräftetrainerin in Wirtschafts- und Nonprofitorganisationen. 